\title{
Progress on Understanding Transcriptional Regulation of Chloroplast Development in Fleshy Fruit
}

\author{
Ting Jia ${ }^{1,2}$, Yuting Cheng ${ }^{3}$, Imran Khan ${ }^{3}$, Xuan Zhao ${ }^{3}$, Tongyu $\mathrm{Gu}^{3}$ and Xueyun $\mathrm{Hu}^{1,3, * \mathbb{C}}$ \\ 1 Key Laboratory of Plant Functional Genomics of the Ministry of Education, Yangzhou University, \\ Yangzhou 225009, China; tingj2012@yzu.edu.cn \\ 2 Joint International Research Laboratory of Agriculture and Agri-Product Safety of the Ministry of Education \\ of China, Yangzhou University, Yangzhou 225009, China \\ 3 College of Bioscience and Biotechnology, Yangzhou University, Yangzhou 225009, China; \\ dx120200180@yzu.edu.cn (Y.C.); dh18006@yzu.edu.cn (I.K.); mx120190786@yzu.edu.cn (X.Z.); \\ mz120191171@yzu.edu.cn (T.G.) \\ * Correspondence: xyhulab@yzu.edu.cn
}

Received: 31 August 2020; Accepted: 17 September 2020; Published: 22 September 2020

\begin{abstract}
Edible fleshy fruits are important food sources in the human diet. Their yield and nutritional quality have long been considered as breeding targets for improvement. Various developing fleshy fruits with functional chloroplasts are capable of photosynthesis and contribute to fruit photosynthate, leading to the accumulation of metabolites associated with nutritional quality in ripe fruit. Although tomato high-pigment mutants with dark-green fruits have been isolated for more than 100 years, our understanding of the mechanism of chloroplast development in fleshy fruit remain poor. During the past few years, several transcription factors that regulate chloroplast development in fleshy fruit were identified through map-based cloning. In addition, substantial progress has been made in elucidating the mechanisms that how these transcription factors regulate chloroplast development. This review provides a summary and update on this progress, with a framework for further investigations of the multifaceted and hierarchical regulation of chloroplast development in fleshy fruit.
\end{abstract}

Keywords: fleshy fruit; chloroplast development; chromoplast; nutritional quality; pigment

\section{Introduction}

Chloroplasts are essential organelles for converting light energy into chemical energy in plant and algal cells. They are not only vital for photosynthesis but also play a pivotal role in plant primary and secondary metabolism, such as the metabolism of amino acids, fatty acids, and phytohormones [1,2]. Given the importance of plant products to human survival, photosynthesis and the biogenesis of chloroplasts have received intensive investigation. In land plants, chloroplasts develop from proplastids that are present in developing meristems, however, chloroplast development are throughout all stages of plant growth [3,4]. For example, within a given Arabidopsis leaf, chloroplast developmental gradients can be observed from the base to the tip and from the margin to the midrib [3,5]. In this review, we also consider that chloroplast division is an integral part of normal chloroplast development, as it was suggested by Pyke [6]. To optimize photosynthesis to varying light regimes, plants have developed a complex and highly regulated chloroplast development process, which may be governed by light signaling, retrograde chloroplast to nucleus signaling, cell-specific chloroplast development, and photosynthetic acclimation to the light environment, as reviewed by Bapat et al. [7], Pfalz and Pfannschmidt [8] and Pogson et al. [3]. 
Leaves are the major site of photosynthesis for most plants, however, developing fleshy fruits are capable of photosynthesis, and it is estimated that up to $20 \%$ of the total fruit carbohydrate comes from the photosynthesis of immature fleshy fruits in tomato [9], the model plant for fleshy fruits. In addition to photosynthesis, during fruit maturation, chloroplasts develop into chromoplasts, synthesizing nutritional metabolites, such as carotenoids (Figure 1) [10]. The transition from chloroplast to chromoplast is often happened in flowers and fruits, but only a few plant species differentiate chromoplasts in leaves [11,12]. During chloroplast to chromoplast transition, besides the remodeling of the internal plastid structures, the most prominent changes are a degradation of photosynthetic competence and over-accumulation of carotenoid pigments, as it was well reviewed by Egea et al. [13]. Therefore, the manipulation of chloroplast to chromoplast differentiation in plants appears as a very promising strategy for improving the nutritional and health benefits of crops [14,15]. On the other hand, it has been demonstrated that tomato fruits with more active chloroplasts at the premature stage will develop more active chromoplasts at the mature stage [16]. Therefore, enhancing fruit chloroplast development can result in higher nutritional value in mature fleshy fruit [17-19].

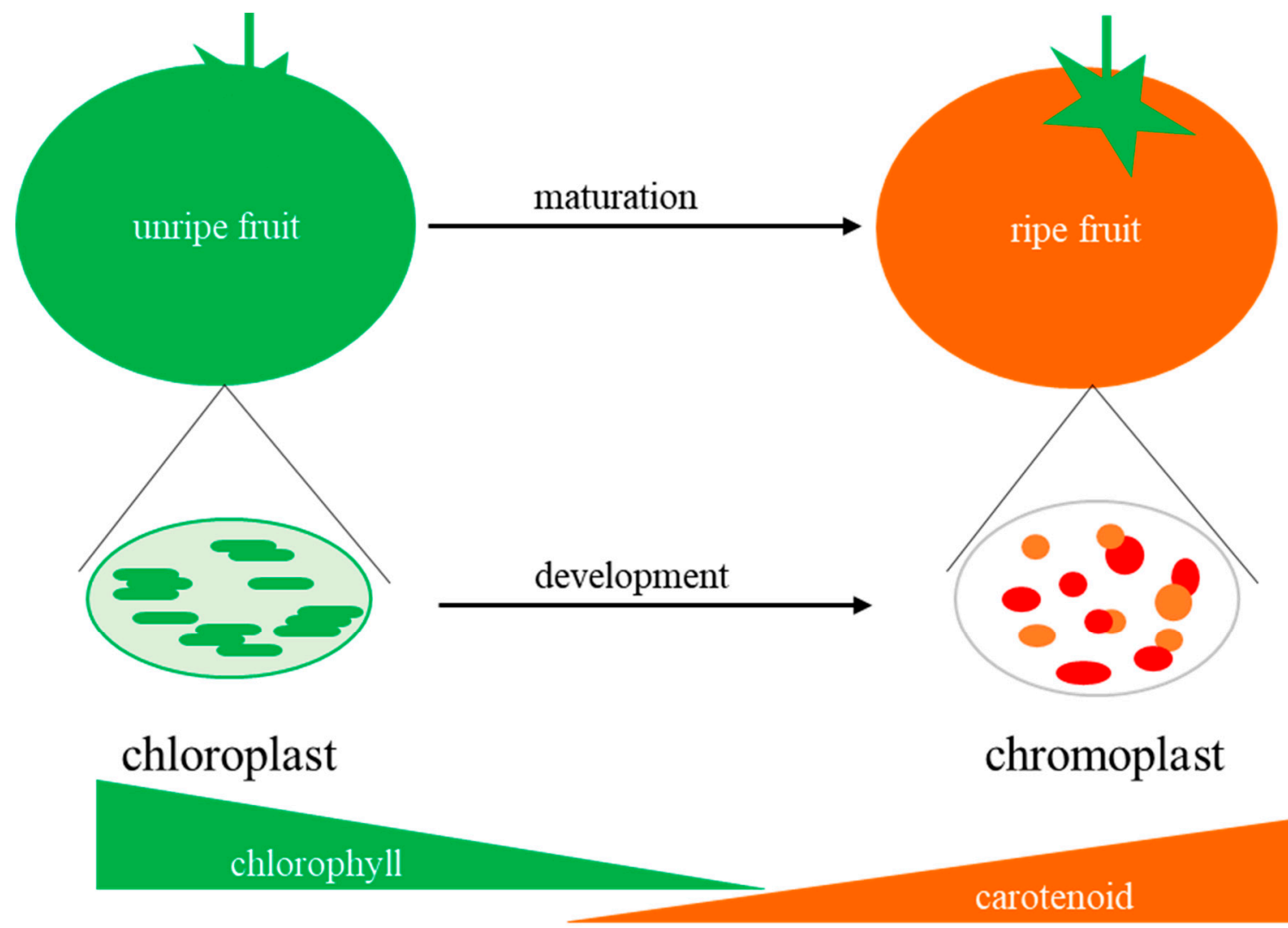

Figure 1. Chloroplast to chromoplast transition during fleshy fruit maturation. During fleshy fruit ripening, chloroplasts undergo changes to form chromoplasts, such as chlorophyll degradation and accumulation of metabolites (such as carotenoids) that contribute to nutritional quality of ripe fruit.

In general, chloroplast development in fruits shares similar regulatory mechanisms with those found in leaves $[17,20]$. However, it was discovered that some transcription factors have special contribution on the regulation of fruit chloroplast development, while they only have little effect on leaf chloroplast development $[17,21,22]$. In the past few years, understanding the molecular mechanism underlying the fine manipulation of chloroplast development in fleshy fruits has been intensively investigated. In this review, we provide an updated summary of the progress made in elucidating the transcriptional regulation of chloroplast development in fleshy fruits, and highlight major knowledge gaps for future study. Finally, strategies for improving fruit nutritional quality through manipulation of chloroplast development in fruits are proposed. 


\section{Key Transcription Factors that Regulate Chloroplast Development in Fleshy Fruits}

\subsection{GLK2 Is a Well-Studied Master Regulator of Chloroplast Development in Fleshy Fruits}

Map-based cloning of the uniform ripening $(u)$ mutation revealed that $U$ encodes a Golden 2-like MYB transcription factor (GLK2), which is a positive regulator of chloroplast development and pigment accumulation in tomato (Solanum lycopersicum) fruit $[17,23]$. Two GLK genes are expressed in tomato, and the SlGLK2 mRNA predominates in fruit. Increasing the expression of SlGLK2 increases chloroplast number and size, producing homogeneously dark-green fruits with enhanced chlorophyll content [17].

Previously, it was reported that GLK transcription factors are conserved in plants and positively regulate chloroplast development in several land plants [24]. In Arabidopsis, there are also two GLK genes (AtGLK1 and AtGLK2), which function redundantly to regulate chloroplast biogenesis in different plant organs. For example, in siliques, AtGLK2 transcripts predominate, while AtGLK1 transcripts do not accumulate; therefore, AtGLK2 knockout mutants exhibit pale-green siliques [24]. By targeting the promoters of a suite of genes associated with photosystem biogenesis and chlorophyll biosynthesis, Arabidopsis GLKs coordinate the transcription of these genes, suggesting that GLKs function in positively regulating chloroplast development [25]. Constitutive overexpression of AtGLK1 and AtGLK2 in tomato increased both the number and size of green fruit chloroplasts and promoted the accumulation and development of grana thylakoids, resulting in increased soluble solids and carotenoids in ripe fruit, for better nutritional quality, while no obvious alterations of leaf chlorophyll or chloroplasts were observed [17]. In pepper (Capsicum annuum L.), it was reported that CaGLK2 is a major quantitative trait locus controlling the natural variation in chlorophyll content and chloroplast compartment size in immature fruit [26]. Heterologous expression of the kiwifruit (Actinidia chinensis) GLK2 homologue in tomato resulted in dark-green immature fruit with increased chloroplast number and size (with enhanced thickness of thylakoid grana stacks) and ripened fruit with elevated contents of carotenoids and sugars [27]. Thus, GLK2 is a widely conserved master transcription factors, which possess specific contribution on the regulation of chloroplast development in fleshy fruits.

\subsection{APRR2-Like Transcription Factors Regulate Chloroplast Development in Fleshy Fruits}

Another transcription factor, ARABIDOPSIS PSEUDO RESPONSE REGULATOR2-LIKE (APRR2-Like) was identified by neural network inference analysis in tomato [18]. The sequence of SIAPRR2-Like has strong homology to that of APRR2, related to but distinct from SIGLK2 [24]. SIAPRR2-Like was upregulated at the breaker stage in wild-type tomato fruits, whilst SIAPRR2-Like overexpressing plants showed increased plastid number and area, higher chlorophyll content in green unripe fruits and elevated carotenoid content in red ripe fruits. However, although the level of APRR2-Like expression in the leaves of the transgenic lines was substantially higher than in the wild-type, the leaf of both transgenic and wild-type had similar total chlorophyll levels. Unlike SIGLK2, SIAPRR2-Like was suggested to play a role in the ripening process, but the underlying mechanism requires further investigation [18]. In the same report, a putative orthologue of the SlAPRR2-Like gene in sweet pepper (Capsicum annuum L.) was found to be associated with pigment accumulation in fruit tissues [18]. Recently, map-based cloning identified that a single-base insertion in the APRR2-Like gene leading to a premature stop codon is responsible for the white immature fruit colour in cucumber (Cucumis satious L.) [28]. More recently, it was reported that the APRR2 gene regulates pigment accumulation in melon and watermelon [29]. Taken together, these studies indicate that the APRR2-Like gene has a conserved function in promoting fruit pigment accumulation and chloroplast development in flowering plants. Thus far, the underlying molecular mechanism of action of APRR2 is unclear.

Since GLK2 is not required for the elevated pigment phenotype of APRR2-Like overexpression lines and the expression of GLK1 was unchanged in APRR2-Like overexpressing plants [18,30], it was suggested that APRR2-Like is unlikely to regulate chloroplast development by mediating the expression of GLKs. On the other hand, APRR2-Like gene expression is not changed in GLK2 overexpressing plants [17]. Although the relationship between GLKs and APRR2-Like needs further investigation, 
it was suggested that APRR2-Like and GLK2 act independently as key transcription factors to directly activate genes involved in fruit chloroplast development [21].

\subsection{TKN2 and TKN4 Regulate the Expression of GLK2 and APRR2}

TKN2 and TKN4, which belong to the class I KNOTTED-LIKE HOMEODOMAIN (KNOX) gene family, were identified to play specific roles in chloroplast development in fruit but not in leaves [21]. Map-based cloning identified a point mutation in TKN4 response to a mutant lacks the green shoulder of developing fruit. The development of chloroplast is impaired, with a reduction in size and in the number of thylakoids per granum in the fruit shoulder of TKN4 unexpressed mutant [21] A dominant gain-of-function mutation that results in ectopic expression of TKN2 is characterized by increased chloroplast number, higher chlorophyll content and larger size of chloroplast in tomato fruits. It is striking that these phenotypes resemble over-expression lines of SlGLK2 and SIAPRR2-Like. Further investigation in tomato showed that TKN2 promotes chloroplast development in fleshy fruit by regulating both SIGLK2 and SIAPRR2-Like expression, whereas TKN4 only regulates the expression of SlGLK2 [21]. It was also found that TKN2 and TKN4 regulate the latitudinal gradient of expression of GLK2 and APRR2-Like across developing tomato fruit, correlating with a gradient in chloroplast development [21].

\subsection{Other Transcription Factors Regulate Chloroplast Development in Fruit}

2.4.1. BELs Negatively Regulate Chloroplast Development and Chlorophyll Synthesis in Tomato Fruit Directly and Indirectly

More recently, seven homeodomain-containing transcription factors with specific ripening-associated expression patterns were investigated, and three BEL1-like (BELL) members showed fruit-specific expression in tomato [22]. BEL1-LIKE HOMEODOMAIN11 (SIBEL11) was shown to regulate chloroplast development and chlorophyll synthesis in tomato fruits. When SIBEL11 expression was reduced by RNAi, chlorophyll levels, chloroplast numbers per cell and the number of thylakoids per chloroplast were significantly increased in unripe tomato fruits, and 48 genes involved in chlorophyll biosynthesis, chloroplast development and photosynthesis were upregulated. Among the upregulated genes in SlBEL11-RNAi plants, 22 appear to be potential targets of SIBEL11 [22]. Furthermore, strong evidence showed that S1BEL11 could repress the expression of TKN2, chlorophyll $a / b$ binding protein $(C A B)$ and chlorophyll biosynthesis-related genes encoding for protochlorophyllide reductase $(P O R)$ by directly binding their promoters in green fruits but had no obvious effect in leaves [22]. Moreover, it was found that DEETIOLATED1 (DET1), a negative regulator of photomorphogenesis and chloroplast development in fleshy fruit [31-33], was also repressed in SlBEL11-RNAi plants [22], indicating that SIBEL11 may also regulate chloroplast development through DET1. Most recently, it was reported that slightly darker-green tomato fruit with increased chlorophyll content, the number of thylakoids per granum of chloroplasts, starch, fructose and glucose resulted from downregulation of BEL1-LIKE HOMEODOMAIN4 (SlBL4). Similar to SIBEL11, SIBL4 also directly inhibits some genes that encode enzymes catalyzing chlorophyll biosynthesis and SITKN2 [34]. Therefore, BELs play hierarchical roles in fruit chloroplast development.

\subsubsection{LOL1 Controls Chloroplast Size in a Fruit-Specific Manner}

QTL map-based cloning identified a zinc-finger transcription factor CcLOL1 (LSD ONE LIKE1; CCLOL1) that regulates chlorophyll content by controlling chloroplast size in a fruit-specific manner in pepper (Capsicum chinense) [35]. SILOL1 retains a similar function in tomato fruit [35], knockout of tomato SILOL1 results in light-green immature fruit with reduced chloroplast size and number. LOL1 was initially identified as a positive regulator of programmed cell death in Arabidopsis [36]. Its rice (Oryza sativa L.) homolog, termed OsLSD1, also control programmed cell death. In addition, overexpressing OsLSD1 resulted in an increase of chlorophyll content in the shoot [37]. However, 
knockout of CCLOL1 or tomato SILOL1 had no significant effect on leaf chlorophyll content [35]. The mechanism by which LOL1 regulates chloroplast development in fleshy fruit is not well known, only transcriptome analysis revealed that genes involved in light harvesting, chlorophyll biosynthesis and $R b c$ genes (encoding ribulose bisphosphate carboxylase subunits) were significantly reduced in CCLOL1 mutated bulk, suggesting the possible involvement of LOL1 in regulation of carbon fixation in the fruit [35].

\section{Light Signaling Pathway Involved in the Transcriptional Regulation of Chloroplast Development in Fleshy Fruits}

In 1917, five photomorphogenic mutants carrying monogenic recessive high-pigment (hp-1, $h p-1^{W}, h p-2, h p-2^{j}$, and $\left.h p-2^{d g}\right)$ mutations were reported [38]. These mutants were characterized by exaggerated light responsiveness and intense fruit pigmentation in tomato, as extensively reviewed [39]. Characteristic phenotypes include dark-green fruits, resulting from increased plastid compartment size, increased chloroplast number, and higher chlorophyll content at pre-ripening stages [31,40-42]. Later, $h p-1$ and $h p-1^{W}$ were mapped to the HP-1 gene, which is located on chromosome 2 [43], while $h p-2$, $h p-2^{j}$, and $h p-2^{d g}$ were found to be allelic and were mapped to the HP-2 gene located on chromosome 1 [44]. Furthermore, cloning of the HP-1 gene revealed that it is the tomato homologue of the Arabidopsis thaliana gene encoding UV-DAMAGED DNA BINDING PROTEIN1 (DDB1) [39,41].

The HP-2 gene encodes the tomato homologue of Arabidopsis DEETIOLATED1 (DET1) [31,32], a negative regulator of photomorphogenesis [33]. In fact, complex purification experiments has shown that DET1 interacts with the DDB1 protein [45]. They form a complex with Cullin4 (CUL4), a ubiquitin-conjugating E3 ligase, targeting proteins for proteolysis in Arabidopsis [46,47]. Therefore, it was suggested that the CUL4-DDB1-DET1 complex participates in chloroplast development and secondary metabolism in tomato fruits [48]. Research results showed that constitutive downregulation of SICUL4 resulted in greater plastid number and higher chlorophyll in unripe fruits and higher carotenoid in ripe fruits, similar to the phenotypes of $h p 1$ mutant and SIDDB1-RNAi plants [48]. Later, it was uncovered that SIGLK2 is a substrate of the CUL4-DDB1-DET1 ubiquitin ligase complex for proteasomal degradation [49].

The CUL4-DDB1-DET1 complex plays a vital role in regulating plant photomorphogenesis, suggesting that the light signal transduction pathway acts an important role in regulating chloroplast development in immature fleshy fruits. To test this hypothesis, LeHY5, the tomato homologue of ELONGATED HYPOCOTYL 5 (HY5) and LeCOP1LIKE, the tomato homologue of CONSTITUTIVELY PHOTOMORPHOGENIC 1 (COP1), which encode positive and negative regulators of light signaling, respectively, were knocked down by RNAi in tomato [41]. LeHY5-RNAi plants showed lighter green leaves and lighter green immature fruits with reduction of thylakoid organization and abundance in comparison to wild-type. In contrast, LeCOP1LIKE-RNAi plants exhibited significantly higher chlorophyll content in the leaf and immature fruit [41]. Furthermore, it was discovered that ripe fruits from $h p-1, h p-2$ and LeCOP1LIKE-RNAi lines had increased total carotenoid levels, whereas ripe fruits of LeHY5-deficient plants possessed less carotenoid than the wild-type [41-43,50]. It was reported that COP1 and SPA (SUPPRESSOR OF PHYA) form a complex together with DDB1-CUL4, targeting HY5 for ubiquitination and degradation [51]. HY5, a master regulator that modulates light-regulated seedling photomorphogenic development, binds to the promoters of almost one-third of Arabidopsis genes [52]. HY5 directly regulates photosynthesis-related genes, such as CAB1 and RbcS1A (encoding $\mathrm{Rbc}$ small chain 1A) [52]. It was also demonstrated that HY5 induces the expression of many genes that are related to root greening by binding to G-box elements (CACGTG) in their promoters [53]. The primary G-box elements for HY5 binding are also highly represented in the promoter of GLK2, but intriguingly not GLK1; therefore, GLK2 is also a putative target of HY5 [25,52]. Moreover, it was found that overexpressing GLKs can only partly induce root greening in the absence of HY5, suggesting that HY5 plays an important role in GLK functions in the root [53]. However, GLK2 has not yet been 
demonstrated to be a direct transcriptional target of HY5, and thus the relationship between HY5 and GLK2 requires further investigation.

Recently, researchers discovered a protein containing a methyl-CpG-binding domain (MBD) in tomato, which was named SIMBD5, is a close homologue of Arabidopsis AtMBD5. The relative expression level of SIMBD5 is remarkably high in fruit, while it is low in leaves. Overexpressing SlMBD5 resulted in dark-green leaves and immature fruits [54]. As in the dark-green fruits of $h p 1$, the number and size of chloroplasts were increased, the chloroplast total area per cell was greater in the outer pericarp of immature green fruits, and levels of lycopene and $\beta$-carotenoids were higher in the ripe fruits of SIMBD5-overexpressing lines in comparison to wild-type [54]. Repressing SIMBD5 by RNAi did not obviously change the phenotype from wild-type, suggesting that SIMBD5 is dispensable for fruit chloroplast development. Further analysis showed that SIMBD5 physically interacts with SIDDB1, and it also interacts with CUL4, DET1, RING-BOX 1a(RBX1a) and RBX1b, supporting the idea that SIMBD5 is functionally linked to the known CUL4-DDB1-based complex [54]. The expression of SIGLK2 was increased in the immature fruits of SIMBD5-overexpressing lines and hp1 mutants, suggesting that the physical interaction between SIMBD5 and DDB1-based complex mediates the transcriptional activities of downstream genes, although the mechanism is still largely unknown [54]. Furthermore, yeast two-hybrid and co-immunoprecipitation assays suggested that SIMBD5 also interacts with the SIGLK2 protein in vivo [54]. SIMBD5 may affect SIGLK2 degradation through the CUL4-DDB1-DET1 ubiquitin ligase complex, which plays a vital role in SIGLK2 degradation by the $26 \mathrm{~S}$ proteasome [49].

Transcript levels of SlGLK2 were elevated in SIMBD5-overexpressing lines, and the expression of SlAPRR2-Like as well as SlGLK2 was considerably enhanced in the $h p 1$ mutant, indicating that the DDB1 signaling pathway plays roles upstream of SIAPRR2-Like and SIGLK2 to regulate chloroplast development [21,55]. The expression of TKN2 and TKN4 was also significantly enhanced in the $h p-1$ mutant. Therefore, it was suggested that TKN2 and TKN4 work downstream of DDB1 and upstream of GLK2 and APRR2 to regulate fruit chloroplast development in tomato [21]. Further investigation is needed to determine whether TKN2 and TKN4 bind directly to the regulatory promoter regions of SIGLK2 and SlAPRR2-Like, and to understand how DDB1 regulates the expression of TKN2 and TKN4 [21].

UV (Ultraviolet) RESISTANCE LOCUS8 (UVR8), the unique UV-B photoreceptor in the UV-B signaling pathway, usually exists as a homodimer, but it is easily converted to monomers in response to UV-B irradiation [56]. Monomerized UVR8 interacts with the COP1-SPA protein complex to form the UVR8-COP1-SPA complex, which negatively affects COP1-SPA1 activity while stabilizing and promoting its HY5 activity [57-59]. Thus the UVR8-COP1-SPA complex can control fruit chloroplast development via HY5. In tomato, SIUVR8 is required for the expression of UV-B-induced SIGLK2 in fruits and leaves. Moreover, it was suggested that SIUVR8 also plays a role in promoting chloroplast development by enhancing the accumulation of SIGLK2 protein under UV-B illumination through post-translational regulation [59]. In SlUVR8-overexpressing plants, higher SIUVR8 levels resulted in a larger plastid area per cell and increased chlorophyll content in immature green fruits. In contrast, decreasing the expression of SIUVR8 resulted in smaller plastid areas per cell and lower chlorophyll content in immature green fruits [59]. Furthermore, more starch and carotenoids accumulated in the fruits of SIUVR8-overexpressing plants [59]. The experiments demonstrated that SIUVR8 plays a vital role in the regulation of chloroplast development in fruits by regulating SIGLK2 and SIHY5. Thus, SIUVR8 is a new candidate to be targeted to enhance both the tolerance to UV-B stress and the nutrient value of fleshy fruits. Therefore, further study is required to demonstrate the detail mechanism of UVR8 on regulating of chloroplast development [59]. 


\section{Linkage between Phytohormone and Chloroplast Development in Fleshy Fruits}

\subsection{ABA Signaling Regulates Chloroplast Development in Fleshy Fruits}

By screening a tomato mutant pool, a mutant named high-pigment $3(h p 3)$ with elevated carotenoid content at all stages of fruit development was identified [16]. The mutation in $h p 3$ corresponds to the gene for zeaxanthin epoxidase (ZEP), a rate-limiting enzyme that converts zeaxanthin to violaxanthin in the pathway for xanthophyll metabolism. The phytohormone abscisic acid (ABA) is derived from xanthophylls, and the $h p 3$ mutant has $75 \%$ lower xanthophyll levels, resulting in ABA-deficiency [16]. Gene expression analysis demonstrated that FtsZ (Filamentous temperature sensitive Z), which encodes a tubulin-like protein involved in plastid division, was significantly increased in $h p 3$, suggesting that plastid division could be elevated in this mutant. Microscopy revealed that the number of plastids per cell of unripe green fruit was increased two-fold in $h p 3-1$ in comparison to wild-type, and the size of plastids was also larger [16]. Although the ABA signaling pathway appears to be involved in regulating chloroplast development in fleshy fruit, the molecular mechanism by which ABA deficiency affects the expression of FtsZ is not known. To further test this hypothesis, two ABA-deficiency mutants, sitiens and flacca, in which ABA biosynthesis is blocked at the ABA-specific aldehyde oxidase and its molybdenum cofactor, respectively were investigated [16]. They were shown to exhibit higher plastid numbers in unripe green fruit cells. Taken together, ABA signaling takes part in chloroplast development in tomato fruits [16].

The CDD (for COP10, DDB1a and DET1) complex interacts with DET1- AND DDB1-ASSOCIATED1 (DDA1) to form the COP10-DET1-DDB1-DDA1 (CDDD) complex, providing substrate specificity for CULLIN4-RING E3 ubiquitin ligase (CRL4), which is responsible for ABA receptor (PYL) degradation [60]. In addition, in rice, it was found that ABA biosynthesis and ABA signaling are modulated by OsDET1 [61]. It seems that ABA signaling regulates fruit chloroplast development downstream of the CDD complex. However, the mechanism by which ABA signaling regulates fruit chloroplast development is not understood. Since APRR1, a protein related to APRR2, has been reported to interact with ABSCISIC ACID INSENSITIVE 3 (ABI3) [62], it was suggested but not yet demonstrated that APRR2-Like interacts with $\mathrm{ABI} 3$ to influence ABA signaling during plastid development and ripening [18].

\subsection{Auxin Signaling Modulates GLK Expression}

Auxin is a well-known phytohormone that modulates plant growth and development mainly through two gene families: the short-lived nuclear protein Aux/IAA family and auxin response factors (ARFs), which regulate auxin-responsive genes on transcriptional level [63]. ARFs can either activate or repress the transcription of auxin-responsive genes by binding to auxin response elements in auxin-responsive gene promoters [63,64]. In Arabidopsis, auxin signaling mediated by IAA14 and ARF7/19 has been described to play an inhibitory role in chloroplast differentiation in roots [53]. In tomato, by reverse genetics approaches, DEVELOPMENTALLY REGULATED 12 (DR12) (now named SlARF4), one of the ARF-like genes, was demonstrated to be a negative regulator of chloroplast development in tomato fruit. The DR12/SlARF4-suppressed lines showed a dark-green immature fruit phenotype with increased chlorophyll and the accumulation of starch, glucose and fructose [65]. SlARF4 expression is high in pericarp tissues of immature fruit; correspondingly, the dark-green phenotype of SIARF4-suppressed lines is restricted to fruit [19]. By quantitative RT-PCR, it was found that the expression of DR12/ARF4 was decreased in the fruits of the $h p 1$ mutant, suggesting that decreased DR12/ARF4 transcription might be one way that plastid compartments are enlarged in immature fruits of $h p 1$ [66]. Recently, it was reported that SIARF4 binds to the promoter of SlGLK2 to suppress its transcription activity [67]. In addition, SlARF4 was found to downregulate SlGLK1 expression in tomato fruits when SlGLK2 was mutated [19]. In another report, SlARF10, another auxin response repressor, targeted the promoter of SIGLK1 and positively regulated SlGLK1 expression in tomato fruits [68]. Overexpression of SlARF10 increased the transcription levels of SlGLK1, SlGLK2, POR, 
chlorophyll binding protein 1 (CBP1) and CBP2 in fruit, whereas its downregulation had the opposite effect [68], which suggests SIARF10 is a positive regulator of fruit chloroplast development. Another ARF, SlARF6A directly bound to the promoters of the $S l G L K 1, C A B$ and $R b c S$ genes and positively regulated the expression of these genes and SIGLK2 both in fruits and leaves in tomato [69]. The sizes of chloroplasts were increased in SIARF6A-overexpressing plants, and the number of chloroplasts was decreased in SIARF6A-downregulated plants. The expression of the SIARF4 and AlARF10 genes was independent of SlARF6A [69], and the expression of SBLIARF4 was independent of SlARF10 [68]. Taken together, these findings show that auxin signaling modulates GLKs expression through different ARFs. Besides ARF4, ARF6A and ARF10, whether other ARFs in auxin signaling pathway involved in the transcriptional regulation of chloroplast development in fleshy fruit awaits further study.

\subsection{Brassinosteroid (BR) Signaling Pathway Modulates GLK Expression}

BR is a phytohormone which involved in plant cell expansion and diverse developmental and physiological processes [70,71]. BR signaling has also been demonstrated to regulate fruit pigment accumulation [72]. BRASSINAZOLE RESISTANT 1 (BZR1) is one of two key transcription factors in BR signaling that directly regulate the expression of many target genes [71]. Ectopic expression of SIBZR1-1D leads to the setting of fruits with a dark-green shoulder at the mature green stage, a similar phenotype to that of $U$ controlled by SIGLK2. Indeed, SIGLK2 expression was upregulated in fruits at the mature green stage when BZR1-1D was overexpressed [72]. Previous research revealed that BZR1, phytochrome-interacting factor 4 (PIF4) and ARF6 activate some of the same target genes, and PIF4 and BZR1 facilitate the binding of ARF6 to its target promoters [73]. It is possible that BZR1 regulates the expression of SIGLK2 through ARF6, as SIARF6A positively regulated the expression of SIGLK2 both in fruits and leaves [69]. Therefore, BR and auxin are interdependent in regulation of fruit chloroplast development. Furthermore information should be obtain to understand how BR signaling pathway modulates chloroplast development in fruit.

\section{Working Models for the Transcriptional Regulation of Fruit Chloroplast Development}

In summary, we propose several models for the transcriptional regulation of fruit chloroplast development based on research progress to date (Figure 2). Basically, GLK2, APRR2 and HY5 are master transcription factors that directly regulate the transcription levels of genes related to chloroplast development, photosynthesis and chlorophyll biosynthesis in fruit. At the transcriptional level, BZR1, ARF6, ARF10, TKN4 and TKN2 positively regulate GLK2, and TKN2 additionally regulates APRR2. ARF4/DR12 negatively regulates the transcription of GLK2 directly by binding its promoter. In addition, BEL11 and BL4 repress TKN2, CAB, POR and other genes to regulate chloroplast development. DDB1 acts upstream of $A R F 4 / D R 12, T K N 4$ and TKN2 to regulate their expression, and MBD5 inhibits DDB1's function.

Additional layers of controls on the transcription factors GLK2 and HY5 occur through post-translational regulation via the CUL4-DDB1-DET1 and COP1-SPA1-DDB1-CUL4 ubiquitin ligase complexes, respectively (Figure 2b,c). MBD5 interacts with the CUL4-DDB1-DET1 complex and inhibits its regulatory function on GLK2. Additionally, MBD5 directly interacts with GLK2 to inhibit its degradation. BEL11 enhances the transcription of DET1, thus leading to formation of the CUL4-DDB1-DET1 complex. UVR8 also plays a role in accumulating GLK2 protein under UV-B illumination through post-translational regulation. UVR8 interacts with the COP1-SPA complex(es) that interferes with the degradation of HY5. In addition, LOL1 represses chloroplast development in fruit through uncertain mechanisms. ARF10 directly targets the GLK1 promoter and transcriptionally activates the expression of GLK1 in tomato fruit, increasing the chlorophyll content of unripe fruits. 


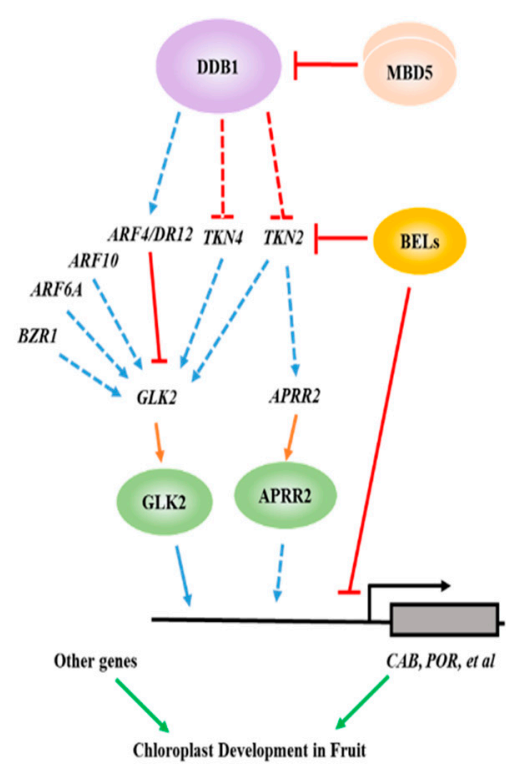

(a)

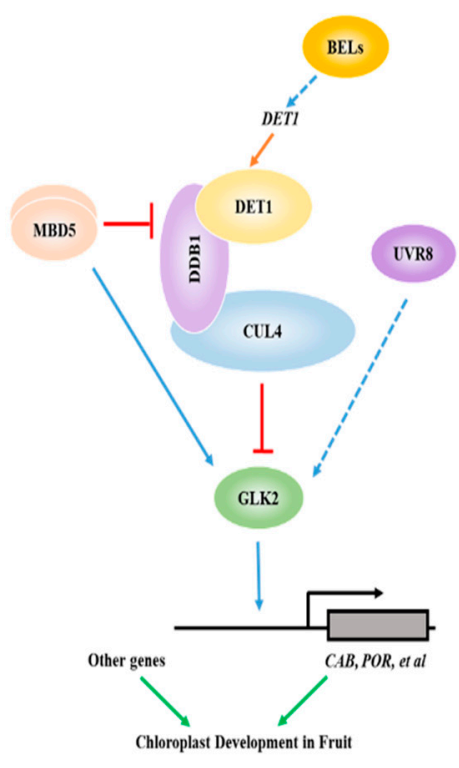

(b)

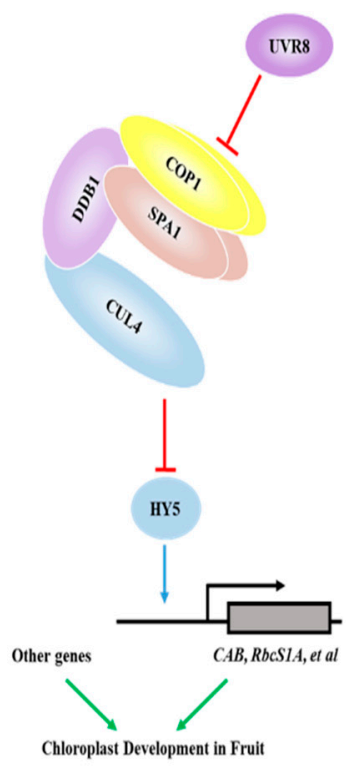

(c)

Figure 2. Models for the transcriptional regulation of chloroplast development in unripe fleshy fruit. (a) The regulation of GLK2 and APRR2 transcription levels to control chloroplast development in fruit. The KNOX genes TKN2 and TKN4 positively affect GLK2 and APRR2 expression to promote chloroplast development. Auxin, via ARFs, regulate the expression of GLK2, whereas brassinosteroids (BRs), via BZR1-1D, promote GLK2 expression (Liu et al., 2014). TKN2 regulates the expression of both GLK2 and $A P R R 2$, whereas TKN4 only regulates the expression of GLK2. DDB1 acts upstream of ARF4/DR12, TKN4 and TKN2 to regulate their expression. The MBD5 dimer blocks DDB1 regulating downstream genes. In addition, BEL11 directly represses the transcription of TKN2, CAB and POR by binding their promoters. (b) Proteasomal degradation of transcriptional regulator GLK2. The CUL4-DDB1-DET1 ubiquitin ligase complex targets GLK2 for proteasomal degradation. MBD5 inhibits this process by interacting with the subunits of this complex. On the other hand, MBD5 directly interacts with GLK2 to inhibit its degradation. BEL11 enhances the transcript levels of DET1, thus helping to form the CUL4-DDB1-DET1 complex. UVR8 also plays a role in accumulating GLK2 protein under UV-B illumination through post-translational regulation. (c) Ubiquitination and degradation of transcriptional regulator HY5. HY5 promotes chloroplast development in fruit, although the mechanism is unclear. COP1-SPA1-DDB1-CUL4, an ubiquitin ligase complex that targets HY5 for ubiquitination and degradation, negatively regulates chloroplast development in fruit. UVR8 interacts with the COP1-SPA complex that interferes with the degradation of HY5. Solid blue and red arrows indicate direct regulation, and the dotted arrows represent indirect regulation or the regulation mechanism is uncertain. Solid orange arrows represents gene transcription and translation. Solid green arrows indicate genes that regulate chloroplast development.

\section{Improving Fruit Phytonutrients by Regulating Fruit Chloroplast Development}

GLK2 is a key transcriptional regulator of chloroplast development and chlorophyll biosynthesis in fruits. In tomato, GLK2 and GLK1 overexpression increased chlorophyll levels in green fruit and increased fructose, glucose, soluble solids and lycopene in red fruit [17]. Upregulation of SlAPRR2-Like increased the plastid number and area, the content of chlorophyll in green unripe fruits, carotenoids in red ripe fruits [18]. Therefore, GLK2 and APRR2-Like are promising candidates to target for improving fruit phytonutrients. Other approaches also can be exploited to improve our current varieties. For example, for a long time, tomato breeders have selected varieties with uniform light green fruit before ripening, for easier maturity determination, however, these fruits also ripen with reduced sugars and carotenoid, compromising flavor of fresh fruits [17]. Since the point mutation in SlGLK2 leads to these traits, in order to recover the benefit traits in current varieties, cross them with 
SIGLK2 highly expressed tomato germplasms, and subsequently multi generation back-cross can be employed. In another way, the fast developing gene editing systems can be used to edit the mutated SlGLK2, thus the function of SlGLK2 can be restored.

Although tomato DET1-impaired mutants ( $h p-2)$ and constitutively DET1-silenced plants showed elevated carotenoids in their mature fruits, severe developmental defects were also observed [31,74]. To avoid the negative effects of $D E T 1$ deficiency on plant growth, one strategy is to specifically repress DET1 in fruits by using fruit-specific promoters combined with RNAi technology. Using this approach, transgenic plants were found to develop dark-green immature fruits that turned deep red at the mature stage [75]. These deep-red fruits displayed significantly higher contents of carotenoids and flavonoids, whereas other parameters of plant growth and fruit quality were largely unchanged. A similar strategy was employed to specifically repress fruit DDB1 expression in tomato, resulting in elevated pigment accumulation in fruits without vegetative growth penalty [48]. These studies give us examples of how to employ genes which are disadvantage to plants growth, but possess bright prospect to promoting chloroplast development in fruit.

\section{Conclusions and Perspectives}

Fleshy fruit is the edible part of many plants. The chloroplast in fleshy fruit is not only the photosynthetic organelle for organic carbon synthesis but also the source of chromoplasts for synthesizing special nutrients, such as the carotenoid lycopene (Figure 1). In this review, we have provided a comprehensive overview of the research progress on the transcriptional regulation of chloroplast development in fleshy fruit. Considering the importance of chloroplast development in fleshy fruit and the distinct regulatory mechanisms of chloroplast development in leaves, it is important to identify more players involved in the transcriptional regulation of chloroplast development in fleshy fruit. For example, recently, it was found that SIELP2L, an elongator complex protein 2-like gene, regulates chloroplast development and chlorophyll accumulation in tomato fruits through epigenetic modification [76]. Furthermore, negative regulators that promote fruit chloroplast development without yield penalty are eagerly to be identified and investigated, thus they can be the ideal targets to be precisely edited by CRISPR/Cas9 in order to create fruits with improved phytonutrients, and these genes edited plants can be considered transgene free that could easily be applicable.

The following step is to demonstrate the mechanisms by which DDB1 regulates the transcription of TKN4, TKN2 and ARF4/DR12, and by which APRR2, HY5, LOL1 and ELP2L regulate fruit-specific chloroplast development need to be clarified. Furthermore, a refined analysis of the relationship between GLK2, APRR2, BEL11, LOL1 and the CDD complex will contribute to more detailed understanding of the mechanism of chloroplast development in fleshy fruit, a mechanism that can be exploited to improve the nutritive value of fruit vegetables and fruits.

Author Contributions: Conceptualization, T.J. and X.H.; writing—original daft preparation, T.J., Y.C., I.K., X.Z., T.G. and X.H.; writing-review and editing, T.J., Y.C., I.K., X.Z., T.G. and X.H.; supervision, T.J. and X.H.; funding acquisition, T.J. and X.H. All authors have read and agreed to the published version of the manuscript.

Funding: This work was supported by the Natural Science Foundation of China (Grant No. 31870265 to X.H., approval date: 16 August 2018), the China Postdoctoral Science Foundation (Grant No. 2018M630608 to X.H., approval date: 4 May 2018), the Natural Science Foundation of Jiangsu Higher Education Institutions of China (Grant No. 18KJB210011 to T.J., approval date: 24 July 2018), the Special Financial Grant from the China Postdoctoral Science Foundation (Grant No. 2019T120467 to X.H., approval date: 14 June 2019), the Innovative and Entrepreneurial Doctor of Jiangsu Province, and "Lvyang Jinfeng" Talents Attracting Plan of Yangzhou.

Acknowledgments: The authors would like to thank Kathrin Schrick (Kansas State University) for critical reading and editing of this manuscript.

Conflicts of Interest: The authors declare no competing interests. 


\section{References}

1. Lopez-Juez, E.; Pyke, K.A. Plastids unleashed: Their development and their integration in plant development. Int. J. Dev. Biol. 2005, 49, 557-577. [CrossRef] [PubMed]

2. Rolland, N.; Bouchnak, I.; Moyet, L.; Salvi, D.; Kuntz, M. The Main Functions of Plastids. In Plastids. Methods in Molecular Biology; Maréchal, E., Ed.; Humana Press: New York, NY, USA, 2018; Volume 1829, pp. $73-85$.

3. Pogson, B.J.; Ganguly, D.; Albrecht-Borth, V. Insights into chloroplast biogenesis and development. Biochim. Biophys. Acta 2015, 1847, 1017-1024. [CrossRef] [PubMed]

4. Chan, K.X.; Phua, S.Y.; Crisp, P.; McQuinn, R.; Pogson, B.J. Learning the languages of the chloroplast: Retrograde signaling and beyond. Annu. Rev. Plant Biol. 2016, 67, 25-53. [CrossRef] [PubMed]

5. Gügel, I.L.; Soll, J. Chloroplast differentiation in the growing leaves of Arabidopsis thaliana. Protoplasma 2017, 254, 1857-1866. [CrossRef]

6. Pyke, K.A. Plastid division and development. Plant Cell 1999, 11, 549-556. [CrossRef]

7. Waters, M.T.; Langdale, J.A. The making of a chloroplast. EMBO J. 2009, 28, 2861-2873. [CrossRef]

8. Pfalz, J.; Pfannschmidt, T. Essential nucleoid proteins in early chloroplast development. Trends Plant Sci. 2013, 18, 186-194. [CrossRef]

9. Hetherington, S.E.; Smillie, R.M.; Davies, W.J. Photosynthetic activities of vegetative and fruiting tissues of tomato. J. Exp. Bot. 1998, 49, 1173-1181. [CrossRef]

10. Cocaliadis, M.F.; Fernández-Muñoz, R.; Pons, C.; Orzaez, D.; Granell, A. Increasing tomato fruit quality by enhancing fruit chloroplast function. A double-edged sword? J. Exp. Bot. 2014, 65, 4589-4598. [CrossRef]

11. Sadali, N.M.; Sowden, R.G.; Ling, Q.; Jarvis, R.P. Differentiation of chromoplasts and other plastids in plants. Plant Cell Rep. 2019, 38, 803-818. [CrossRef]

12. Sun, T.; Yuan, H.; Cao, H.; Yazdani, M.; Tadmor, Y.; Li, L. Carotenoid metabolism in plants: The role of plastids. Mol. Plant 2018, 11, 58-74. [CrossRef]

13. Egea, I.; Barsan, C.; Bian, W.; Purgatto, E.; Latché, A.; Chervin, C.; Bouzayen, M.; Pech, J.C. Chromoplast differentiation: Current status and perspectives. Plant Cell Physiol. 2010, 51, 1601-1611. [CrossRef]

14. Wurtzel, E.T. Changing form and function through carotenoids and synthetic biology. Plant Physiol. 2019, 179, 830-843. [CrossRef] [PubMed]

15. Llorente, B.; Torres-Montilla, S.; Morelli, L.; Florez-Sarasa, I.; Matus, J.T.; Ezquerro, M.; D’Andrea, L.; Houhou, F.; Majer, E.; Picó, B.; et al. Synthetic conversion of leaf chloroplasts into carotenoid-rich plastids reveals mechanistic basis of natural chromoplast development. Proc. Natl. Acad. Sci. USA 2020, 117, 21796-21803. [CrossRef] [PubMed]

16. Galpaz, N.; Wang, Q.; Menda, N.; Zamir, D.; Hirschberg, J. Abscisic acid deficiency in the tomato mutant high-pigment 3 leading to increased plastid number and higher fruit lycopene content. Plant J. 2008, 53, 717-730. [CrossRef] [PubMed]

17. Powell, A.L.T.; Nguyen, C.V.; Hill, T.; Cheng, K.L.; Figueroa-Balderas, R.; Aktas, H.; Ashrafi, H.; Pons, C.; Fernandes-Munoz, R.; Vicente, A.; et al. Uniform ripening encodes a Golden 2-like transcription factor regulating tomato fruit chloroplast development. Science 2012, 336, 1711-1715. [CrossRef]

18. Pan, Y.; Bradley, G.; Pyke, K.; Ball, G.; Lu, C.; Fray, R.; Marshall, A.; Jayasuta, S.; Baxter, C.; van Wijk, R.; et al. Network inference analysis identifies an APRR2-Like gene linked to pigment accumulation in tomato and pepper fruits. Plant Physiol. 2013, 161, 1476-1485. [CrossRef]

19. Sagar, M.; Chervin, C.; Mila, I.; Hao, Y.; Roustan, J.-P.; Benichou, M.; Gibon, Y.; Biais, B.; Maury, P.; Latche, A.; et al. SlARF4, an auxin response factor involved in the control of sugar metabolism during tomato fruit development. Plant Physiol. 2013, 161, 1362-1374. [CrossRef]

20. Matas, A.J.; Yeats, T.H.; Buda, G.J.; Zheng, Y.; Chatterjee, S.; Tohge, T.; Ponnala, L.; Adato, A.; Aharoni, A.; Stark, R.; et al. Tissue- and cell-type specific transcriptome profiling of expanding tomato fruit provides insights into metabolic and regulatory specialization and cuticle formation. Plant Cell 2011, 23, 3893-3910. [CrossRef]

21. Nadakuduti, S.S.; Holdsworth, W.L.; Klein, C.L.; Barry, C.S. KNOX genes influence a gradient of fruit chloroplast development through regulation of GOLDEN2-LIKE expression in tomato. Plant J. 2014, 78, 1022-1033. [CrossRef] 
22. Meng, L.; Fan, Z.; Zhang, Q.; Wang, C.; Gao, Y.; Deng, Y.; Zhu, B.; Zhu, H.; Chen, J.; Shan, W.; et al. BEL1-LIKE HOMEODOMAIN 11 regulates chloroplast development and chlorophyll synthesis in tomato fruit. Plant J. 2018, 94, 1126-1140. [CrossRef] [PubMed]

23. Nguyen, C.V.; Vrebalov, J.T.; Gapper, N.E.; Zheng, Y.; Zhong, S.; Fei, Z.; Giovannoni, J.J. Tomato GOLDEN2-LIKE transcription factors reveal molecular gradients that function during fruit development and ripening. Plant Cell 2014, 26, 585-601. [CrossRef] [PubMed]

24. Fitter, D.W.; Martin, D.J.; Copley, M.J.; Scotland, R.W.; Langdale, J.A. GLK gene pairs regulate chloroplast development in diverse plant species. Plant J. 2002, 31, 713-727. [CrossRef] [PubMed]

25. Waters, M.T.; Wang, P.; Korkaric, M.; Capper, R.G.; Saunders, N.J.; Langdale, J.A. GLK transcription factors coordinate expression of the photosynthetic apparatus in Arabidopsis. Plant Cell 2009, 21, 1109-1128. [CrossRef] [PubMed]

26. Brand, A.; Borovsky, Y.; Hill, T.; Afnan, K.; Rahman, K.A.A.; Bellalou, A.; Van Deynze, A.; Paran, I. CaGLK2 regulates natural variation of chlorophyll content and fruit color in pepper fruit. Theor. Appl. Genet. 2014, 127, 2139-2148. [CrossRef]

27. Li, G.; Chen, D.; Tang, X.; Liu, Y. Heterologous expression of kiwifruit (Actinidia chinensis) GOLDEN2-LIKE homolog elevates chloroplast level and nutritional quality in tomato (Solanum lycopersicum). Planta 2018, 247, 1351-1362. [CrossRef]

28. Liu, H.; Jiao, J.; Liang, X.; Liu, J.; Meng, H.; Chen, S.; Li, Y.; Cheng, Z. Map-based cloning, identification and characterization of the $w$ gene controlling white immature fruit color in cucumber (Cucumis sativus L.). Theor. Appl. Genet. 2016, 129, 1247-1256. [CrossRef]

29. Oren, E.; Tzuri, G.; Vexler, L.; Dafna, A.; Meir, A.; Faigenboim, A.; Kenigswald, M.; Portnoy, V.; Schaffer, A.A.; Levi, A.; et al. The multi-allelic APRR2 gene is associated with fruit pigment accumulation in melon and watermelon. J. Exp. Bot. 2019, 70, 3781-3794. [CrossRef]

30. Carvalho, R.F.; Campos, M.L.; Pino, L.E.; Crestana, S.L.; Zsögön, A.; Lima, J.E.; Benedito, V.A.; Peres, L.E.P. Convergence of developmental mutants into a single tomato model system: "Micro-Tom" as an effective toolkit for plant development research. Plant Methods 2011, 7, e18. [CrossRef]

31. Mustilli, A.C.; Fenzi, F.; Ciliento, R.; Alfano, F.; Bowler, C. Phenotype of the tomato high pigment-2 mutant is caused by a mutation in the tomato homolog of DEETIOLATED1. Plant Cell 1999, 11, 145-158. [CrossRef]

32. Levin, I.; Frankel, P.; Gilboa, N.; Tanny, S.; Lalazar, A. The tomato dark green mutation is a novel allele of the tomato homolog of the DEETIOLATED1 gene. Theor. Appl. Genet. 2003, 106, 454-460. [CrossRef] [PubMed]

33. Lau, O.S.; Deng, X.W. The photomorphogenic repressors COP1 and DET1: 20 years later. Trends Plant Sci. 2012, 17, 584-593. [CrossRef] [PubMed]

34. Yan, F.; Gao, Y.; Pang, X.; Xu, X.; Zhu, N.; Chan, H.; Hu, G.; Wu, M.; Yuan, Y.; Li, H.; et al. SlBL4 regulates chlorophyll accumulation, chloroplast development and cell wall metabolism in tomato fruit. J. Exp. Bot. 2020, 71, 5549-5561. [CrossRef]

35. Borovsky, Y.; Monsonego, N.; Mohan, V.; Shabtai, S.; Kamara, I.; Faigenboim, A.; Hill, T.; Chen, S.; Stoffel, K.; Van Deynze, A.; et al. The zinc-finger transcription factor CcLOL1 controls chloroplast development and immature pepper fruit color in Capsicum chinense and its function is conserved in tomato. Plant J. 2019, 99, 41-55. [CrossRef] [PubMed]

36. Epple, P.; Mack, A.A.; Morris, V.R.F.; Dangl, J.L. Antagonistic control of oxidative stress-induced cell death in Arabidopsis by two related, plant-specific zinc finger proteins. Proc. Natl. Acad. Sci. USA 2003, 100, 6831-6836. [CrossRef]

37. Wang, L.; Pei, Z.; Tian, Y.; He, C. OsLSD1, a rice zinc finger protein, regulates programmed cell death and callus differentiation. Mol. Plant Microbe Interact. 2005, 18, 375-384. [CrossRef]

38. Reynard, G.B. Origin of the Webb Special (Black Queen) tomato. Rep. Tomato Genet. Coop. 1956, 6, 22.

39. Lieberman, M.; Segev, O.; Gilboa, N.; Lalazar, A.; Levin, I. The tomato homolog of the gene encoding UV-damaged DNA binding protein 1 (DDB1) underlined as the gene that causes the high pigment-1 mutant phenotype. Theor. Appl. Genet. 2004, 108, 1574-1581. [CrossRef]

40. Kerckhoffs, L.H.J.; De Groot, N.A.M.A.; Van Tuinen, A.; Schreuder, M.E.L.; Nagatani, A.; Koornneef, M.; Kendrick, R.E. Physiological characterization of exaggerated-photoresponse mutants of tomato. J. Plant Physiol. 1997, 150, 578-587. [CrossRef] 
41. Liu, Y.; Roof, S.; Ye, Z.; Barry, C.; van Tuinen, A.; Vrebalov, J.; Bowler, C.; Giovannoni, J. Manipulation of light signal transduction as a means of modifying fruit nutritional quality in tomato. Proc. Natl. Acad. Sci. USA 2004, 101, 9897-9902. [CrossRef]

42. Kolotilin, I.; Koltai, H.; Tadmor, Y.; Bar-Or, C.; Reuveni, M.; Meir, A.; Nahon, S.; Shlomo, H.; Chen, L.; Levin, I. Transcriptional profiling of high pigment-2 ${ }^{d g}$ tomato mutant links early fruit plastid biogenesis with its overproduction of phytonutrients. Plant Physiol. 2007, 145, 389-401. [CrossRef] [PubMed]

43. Yen, H.C.; Shelton, B.A.; Howard, L.R.; Lee, S.; Vrebalov, J.; Giovannoni, J.J. The tomato high-pigment (hp) locus maps to chromosome 2 and influences plastome copy number and fruit quality. Theor. Appl. Genet. 1997, 95, 1069-1079. [CrossRef]

44. van Tuinen, A.; Cordonnier-Pratt, M.M.; Pratt, L.H.; Verkerk, R.; Zabel, P.; Koornneef, M. The mapping of phytochrome genes and photomorphogenic mutants of tomato. Theor. Appl. Genet. 1997, 94, 115-122. [CrossRef] [PubMed]

45. Schroeder, D.F.; Gahrtz, M.; Maxwell, B.B.; Cook, R.K.; Kan, J.M.; Alonso, J.M.; Ecker, J.R.; Chory, J. De-etiolated 1 and Damaged DNA Binding Protein 1 interact to regulate Arabidopsis photomorphogenesis. Curr. Biol. 2002, 12, 1462-1472. [CrossRef]

46. Bernhardt, A.; Lechner, E.; Hano, P.; Schade, V.; Dieterle, M.; Anders, M.; Dubin, M.J.; Benvenuto, G.; Bowler, C.; Genschik, P.; et al. CUL4 associates with DDB1 and DET1 and its downregulation affects diverse aspects of development in Arabidopsis thaliana. Plant J. 2006, 47, 591-603. [CrossRef]

47. Chen, H.; Shen, Y.; Tang, X.; Yu, L.; Wang, J.; Guo, L.; Zhang, Y. Arabidopsis CULLIN4 forms an E3 ubiquitin ligase with RBX1 and the CDD complex in mediating light control of development. Plant Cell 2006, 18, 1991-2004. [CrossRef]

48. Wang, S.; Liu, J.; Feng, Y.; Niu, X.; Giovannoni, J.; Liu, Y. Altered plastid levels and potential for improved fruit nutrient content by downregulation of the tomato DDB1-interacting protein CUL4. Plant J. 2008, 55, 89-103. [CrossRef]

49. Tang, X.; Miao, M.; Niu, X.; Zhang, D.; Cao, X.; Jin, X.; Zhu, Y.; Fan, Y.; Wang, H.; Liu, Y.; et al. Ubiquitin-conjugated degradation of golden 2-like transcription factor is mediated by CUL4-DDB1-based E3 ligase complex in tomato. New Phytol. 2016, 209, 1028-1039. [CrossRef]

50. Bino, R.J.; De Vos, C.H.R.; Lieberman, M.; Hall, R.D.; Bovy, A.; Jonker, H.H.; Tikunov, Y.; Lommen, A.; Moco, S.; Levin, I. The light-hyperresponsive high pigment- $2^{d g}$ mutation of tomato: Alterations in the fruit metabolome. New Phytol. 2005, 166, 427-438. [CrossRef]

51. Osterlund, M.T.; Hardtke, C.S.; Ning, W.; Deng, X.W. Targeted destabilization of HY5 during light-regulated development of Arabidopsis. Nature 2000, 405, 462-466. [CrossRef]

52. Lee, J.; He, K.; Stolc, V.; Lee, H.; Figueroa, P.; Gao, Y.; Tongprasit, W.; Zhao, H.; Lee, I.; Xing, W.D. Analysis of transcription factor HY5 genomic binding sites revealed its hierarchical role in light regulation of development. Plant Cell 2007, 19, 731-749. [CrossRef] [PubMed]

53. Kobayashi, K.; Baba, S.; Obayashi, T.; Sato, M.; Toyooka, K.; Keranen, M.; Aro, E.-M.; Fukaki, H.; Ohta, H.; Sugimoto, K.; et al. Regulation of root greening by light and auxin/cytokinin signaling in Arabidopsis. Plant Cell 2012, 24, 1081-1095. [CrossRef] [PubMed]

54. Li, Y.; Deng, H.; Miao, M.; Li, H.; Huang, S.; Wang, S.; Liu, Y. Tomato MBD5, a methyl CpG binding domain protein, physically interacting with UV-damaged DNA binding protein-1, functions in multiple processes. New Phytol. 2016, 210, 208-226. [CrossRef]

55. Rohrmann, J.; Tohge, T.; Alba, R.; Osorio, S.; Caldana, C.; McQuinn, R.; Arvidsson, S.; Van Der Merwe, M.J.; Riaño-Pachón, D.M.; Mueller-Roeber, B.; et al. Combined transcription factor profiling, microarray analysis and metabolite profiling reveals the transcriptional control of metabolic shifts occurring during tomato fruit development. Plant J. 2011, 68, 999-1013. [CrossRef]

56. Rizzini, L.; Favory, J.J.; Cloix, C.; Faggionato, D.; O’Hara, A.; Kaiserli, E.; Baumeister, R.; Schäfer, E.; Nagy, F.; Jenkins, G.I.; et al. Perception of UV-B by the Arabidopsis UVR8 protein. Science 2011, 332, 103-106. [CrossRef] [PubMed]

57. Huang, X.; Ouyang, X.; Yang, P.; Sun, O.; Chen, L.; Wei, N.; Wang, X. Conversion from CUL4-based COP1-SPA E3 apparatus to UVR8-COP1-SPA complexes underlies a distinct biochemical function of COP1 under UV-B. Proc. Natl. Acad. Sci. USA 2013, 110, 16669-16674. [CrossRef]

58. Yin, R.; Arongaus, A.B.; Binkert, M.; Ulm, R. Two distinct domains of the UVR8 photoreceptor interact with COP1 to initiate UV-B signaling in Arabidopsis. Plant Cell 2015, 27, 202-213. [CrossRef] 
59. Li, H.; Li, Y.; Deng, H.; Sun, X.; Wang, A.; Tang, X.; Gao, Y.; Zhang, N.; Wang, L.; Yang, S.; et al. Tomato UV-B receptor SIUVR8 mediates plant acclimation to UV-B radiation and enhances fruit chloroplast development via regulating SIGLK2. Sci. Rep. 2018, 8, e6097. [CrossRef]

60. Irigoyen, M.L.; Iniesto, E.; Rodriguez, L.; Puga, M.I.; Yanagawa, Y.; Pick, E.; Strickland, E.; Paz-Ares, J.; Wei, N.; De Jaeger, G.; et al. Targeted degradation of abscisic acid receptors is mediated by the ubiquitin ligase substrate adaptor DDA1 in Arabidopsis. Plant Cell 2014, 26, 712-728. [CrossRef]

61. Zang, G.; Zou, H.; Zhang, Y.; Xiang, Z.; Huang, J.; Luo, L.; Wang, C.; Lei, K.; Li, X.; Song, D.; et al. The de-etiolated 1 homolog of Arabidopsis modulates the ABA signaling pathway and ABA biosynthesis in rice. Plant Physiol. 2016, 171, 1259-1276.

62. Kurup, S.; Jones, H.D.; Holdsworth, M.J. Interactions of the developmental regulator ABI3 with proteins identified from developing Arabidopsis seeds. Plant J. 2000, 21, 143-155. [CrossRef] [PubMed]

63. Chandler, J.W. Auxin response factors. Plant Cell. Environ. 2016, 39, 1014-1028. [CrossRef] [PubMed]

64. Roosjen, M.; Paque, S.; Weijers, D. Auxin Response Factors: Output control in auxin biology. J. Exp. Bot. 2018, 69, 179-188. [CrossRef] [PubMed]

65. Jones, B.; Frasse, P.; Olmos, E.; Zegzouti, H.; Li, Z.G.; Latché, A.; Pech, J.C.; Bouzayen, M. Down-regulation of DR12, an auxin-response-factor homolog, in the tomato results in a pleiotropic phenotype including dark green and blotchy ripening fruit. Plant J. 2002, 32, 603-613. [CrossRef]

66. Tang, X.; Tang, Z.; Huang, S.; Liu, J.; Liu, J.; Shi, W.; Tian, X.; Li, Y.; Zhang, D.; Yang, J.; et al. Whole transcriptome sequencing reveals genes involved in plastid/chloroplast division and development are regulated by the HP1/DDB1 at an early stage of tomato fruit development. Planta 2013, 238, 923-936. [CrossRef] [PubMed]

67. Lupi, A.C.D.; Lira, B.S.; Gramegna, G.; Trench, B.; Alves, F.R.R.; Demarco, D.; Peres, L.E.P.; Purgatto, E.; Freschi, L.; Rossi, M. Solanum lycopersicum GOLDEN 2-LIKE 2 transcription factor affects fruit quality in a light- and auxin-dependent manner. PLoS ONE 2019, 14, 1-22. [CrossRef] [PubMed]

68. Yuan, Y.; Mei, L.; Wu, M.; Wei, W.; Shan, W.; Gong, Z.; Zhang, Q.; Yang, F.; Yan, F.; Zhang, Q.; et al. SlARF10, an auxin response factor, is involved in chlorophyll and sugar accumulation during tomato fruit development. J. Exp. Bot. 2018, 69, 5507-5518. [CrossRef]

69. Yuan, Y.; Xu, X.; Gong, Z.; Tang, Y.; Wu, M.; Yan, F.; Zhang, X.; Zhang, Q.; Yang, F.; Hu, X.; et al. Auxin response factor $6 \mathrm{~A}$ regulates photosynthesis, sugar accumulation, and fruit development in tomato. Hortic. Res. 2019, 6, e85. [CrossRef]

70. Wang, W.; Bai, M.Y.; Wang, Z.Y. The brassinosteroid signaling network-a paradigm of signal integration. Curr. Opin. Plant Biol. 2014, 21, 147-153. [CrossRef]

71. Kim, E.J.; Russinova, E. Brassinosteroid signalling. Curr. Biol. 2020, 30, R294-R298. [CrossRef]

72. Liu, L.; Jia, C.; Zhang, M.; Chen, D.; Chen, S.; Guo, R.; Guo, D.; Wang, Q. Ectopic expression of a BZR1-1D transcription factor in brassinosteroid signalling enhances carotenoid accumulation and fruit quality attributes in tomato. Plant Biotechnol. J. 2014, 12, 105-115. [CrossRef] [PubMed]

73. Oh, E.; Zhu, J.Y.; Bai, M.Y.; Arenhart, R.A.; Sun, Y.; Wang, Z.Y. Cell elongation is regulated through a central circuit of interacting transcription factors in the Arabidopsis hypocotyl. Elife 2014, 3, e03031. [CrossRef] [PubMed]

74. Davuluri, G.R.; van Tuinen, A.; Mustilli, A.C.; Manfredonia, A.; Newman, R.; Burgess, D.; Brummell, D.A.; King, S.R.; Palys, J.; Uhlig, J.; et al. Manipulation of DET1 expression in tomato results in photomorphogenic phenotypes caused by post-transcriptional gene silencing. Plant J. 2004, 40, 344-354. [CrossRef] [PubMed]

75. Davuluri, G.R.; van Tuinen, A.; Fraser, P.D.; Manfredonia, A.; Newman, R.; Burgess, D.; Brummell, D.A.; King, S.R.; Palys, J.; Uhlig, J.; et al. Fruit-specific RNAi-mediated suppression of DET1 enhances carotenoid and flavonoid content in tomatoes. Nat. Biotechnol. 2005, 23, 890-895. [CrossRef] [PubMed]

76. Zhu, M.; Li, Y.; Chen, G.; Ren, L.; Xie, Q.; Zhao, Z.; Hu, Z. Silencing SIELP2L, a tomato Elongator complex protein 2-like gene, inhibits leaf growth, accelerates leaf, sepal senescence, and produces dark-green fruit. Sci. Rep. 2015, 5, e7693. [CrossRef]

(C) 2020 by the authors. Licensee MDPI, Basel, Switzerland. This article is an open access article distributed under the terms and conditions of the Creative Commons Attribution (CC BY) license (http://creativecommons.org/licenses/by/4.0/). 